\title{
SEMI-AUTOMATED TOPOLOGY ADJUSTMENT OF PARTIAL WALL GEOMETRY
}

\author{
M. Bassier *, M. Vergauwen \\ Dept. of Civil Engineering, TC Construction - Geomatics \\ KU Leuven - Faculty of Engineering Technology \\ Ghent, Belgium \\ (maarten.bassier,maarten.vergauwen)@kuleuven.be
}

Commission V, WG V/7 \& Commission IV, WG IV/6

KEY WORDS: Building Information Modeling, Topology, Walls, Building, Point Clouds

\begin{abstract}
:
The reconstruction of Building Information Modeling objects for as-built modeling is currently the subject of ongoing research. A popular method is to extract building information from point cloud data to create a set of parametric objects. The automation of this process is highly desired by the industry but is currently hindered by occlusions, clutter and the complexity of the building geometry. To create an as-built BIM, it is vital to not only accurately reconstruct the building's structure but also to compute the topology between the objects. More specifically, we target the topology of the reconstructed partial wall geometry as this forms the basis for other objects.

In this work, a novel method is presented to automatically adjust the topology of wall geometry in an as-built BIM. We present a semi-automated method that procedurally evaluates the configuration of reconstructed objects and adjusts them to create a more faithful BIM. A wall connection evaluation algorithm is proposed that takes as input the centrelines of partial wall geometry and a set of floor and ceilings mesh segments and outputs the topologically adjusted objects. The method is tested on a variety of scenes and shows promising results to reliably compute the topology of as-built models. The generated geometry is similar to the geometric modification proposed by expert modelers. A key advantage is that the algorithm operates directly in Revit and Rhino and can be used for new models as well as for updating existing models.
\end{abstract}

\section{INTRODUCTION}

The reconstruction of a Building Information Modeling (BIM) of an existing building involves the creation of a set of parametric objects that form a coherent structure according to the built situation. First, the structure of the building is created i.e. the walls and slabs, after which other objects can be added to the model (Tah et al., 2018). In this work, we focus on the walls since the slabs can be semi-automatically generated given the watertight representation of the walls. These objects are created either by extruding the 2D plans of the structure or by designing it based on point cloud data (Gimenez et al., 2015). The latter is preferred as plan information is often inaccurate due to undocumented construction changes. The procedure involves experts to manually locate the points that belong to the walls of the structure and create a logical BIM from these observations. As this is a tedious and time consuming procedure, the uptake of automating the process is enormous for the Architectural Engineering and Construction industry (AEC) (Kavanaugh, 2013, Volk et al., 2014). However, the retrieval of wall geometry from point cloud data has several obstacles. First of all, only a portion of the wall faces of each wall is observed. There are occlusions from furniture and false ceilings but also the connections of the walls cannot be captured with remote sensing. While the geometry of each wall can be reasonably accurately reconstructed from the observations, the topology and the connections of the walls can only be derived from reasoning about the building logic of built structures. It is specifically this topology that is the emphasis of this work. We look to automate the reconstruction of the connections between wall elements given a set of wall observations.

\footnotetext{
${ }^{*}$ Corresponding author
}

The reconstruction of the wall topology is part of what is referred to as the Scan-to-BIM process. It is considered the final step to create a faithful BIM representation of a structure from point cloud data in an unsupervised manner. Scan-to-BIM currently is still a process that is part of ongoing research (Patraucean et al., 2015). Both bottom-up or top-down procedures are proposed (Hichri et al., 2013). The former considers a range of measurements from the site as input and extracts increasingly higher level information from the observations until the intended geometry can be reliably constructed. This is a general approach which relies on building logic to interpret the data. It is often used in projects that do not have access to other reliable sources of information such is the case with insufficiently documented buildings and heritage structures. In contrast, the latter relies on this prior information for the point cloud interpretation and reconstructs a well defined number of objects in a selective manner. This is considered a supervised pattern recognition approach which is commonly used in Scan-vs-BIM (Bosché et al., 2013, Bosché et al., 2014). In this research, we propose a bottom-up method since it is generally applicable (Gimenez et al., 2015). Furthermore, we solely rely on the point cloud and building logic for the point cloud interpretation since the incorporation of e.g. sensor information would make the procedure sensor-dependent.

The topology reconstruction is preceded by a number of steps in the Scan-to-BIM process including the segmentation, classification, clustering and finally the reconstruction of the objects. This research solely discuses the final step and takes as input the partial LOD200 BIM walls from prior work (Bassier et al., 2018b, Bassier, Vergauwen, 2019). The presented method takes these inputs and iteratively computes the most likely connec- 


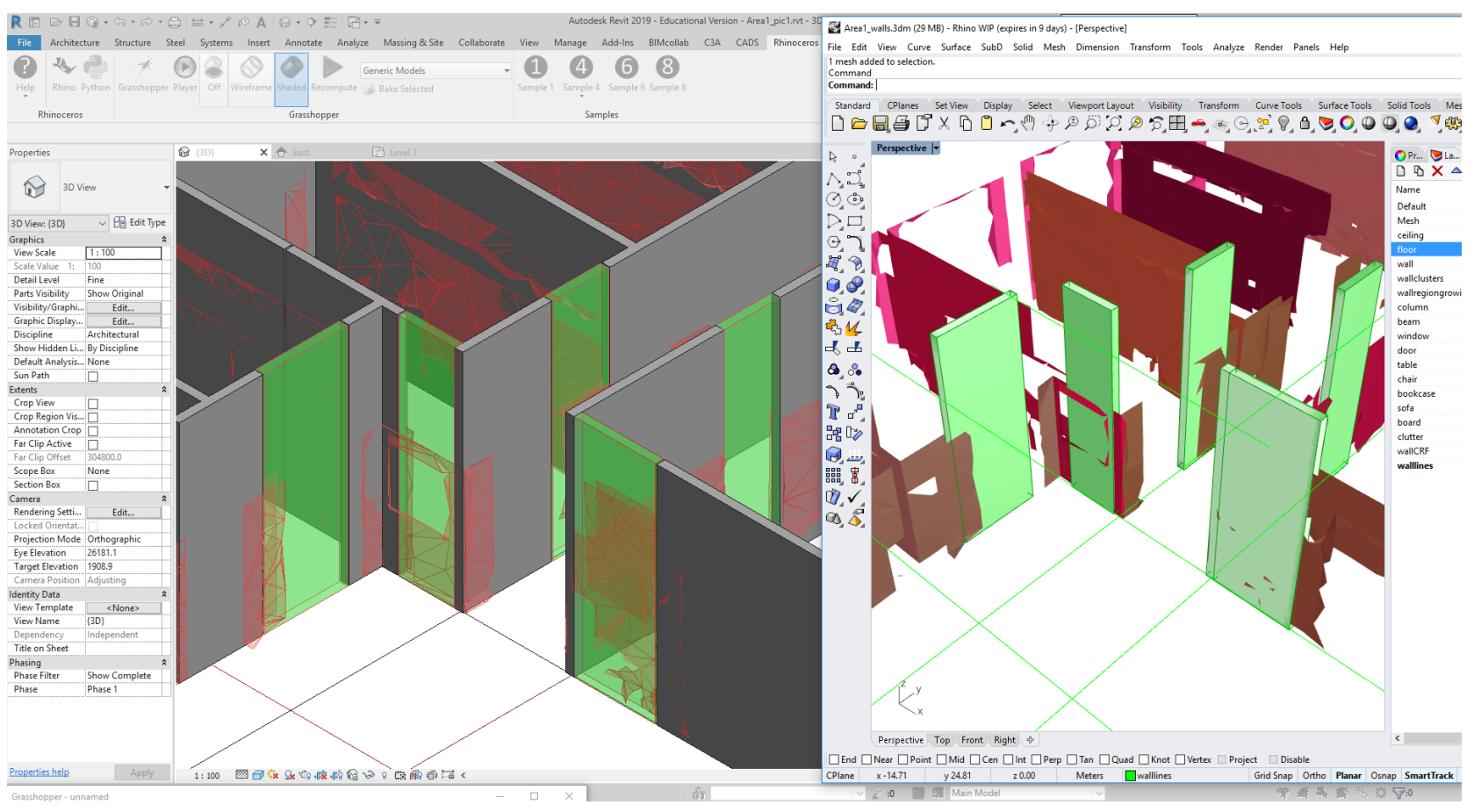

Figure 1. Automated wall topology adjustment using Rhinocommon and Rhino.Inside: (1) The observed wall geometry from remote sensing shown in Rhino, (2) The partial wall geometry is shown in Revit (grey), (3) the wall axes are shown as green lines and (4) the additional wall geometry created to complete the topology is shown in green.

tions. The method differs from existing literature as it deals with both straight edges as well as arcs and polylines. The goal is to compute a watertight model of multi-storey buildings in an automated manner. Furthermore, our approach creates both Revit and Rhino native geometry which ensures data compatibility with a wide range of industry applications.

The remainder of this work is structured as follows. The background and related work is presented in Section 2. In Section 3, the methodology is presented. The test design and experimental results are proposed in Section 4. Finally, the conclusions are presented in Section 5.

\section{BACKGROUND \& RELATED WORK}

The reconstruction of the topology between walls is still the topic of ongoing research (Nguyen, Le, 2013). Both 2D and $3 \mathrm{D}$ methods are proposed along with a variety of inputs. First of all, there are the wall intersection algorithms that compute which set of wall intersections is the most suitable to represent the as-built BIM. For instance, Valero et al. solves the intersections of pre-segmented wall lines to create a closed area (Valero et al., 2012). They retain the first intersection which scores well for single rooms with proper observations as does Xiong et al. (Xiong et al., 2013).

Probably the most commonly used method to create watertight wall geometry is cell decomposition. This is typically a $2 \mathrm{D}$ method that relies on a set of semi-infinite rays to compute the boundaries of a target geometry. First, a set of straight lines is computed based on the observed walls. These can represent the wall faces or the centreline of the wall. Following, this geometry is used to fit a set of semi infinite rays. The rays intersect within the dimensions of the building, thus creating a $2 \mathrm{D}$ cell grid of the structure. Following, the cells are merged together based on some seeding criteria such as the presence of floor or ceiling geometry. The rays at the edge of each cluster subsequently form the boundary of the object's geometry. For instance, Budroni et al. (Budroni, Boehm, 2010) feed their detected wall face planes to a $2 \mathrm{D}$ cell decomposition that retains the set of intersections that encloses their floor geometry. Their method is capable of finding the proper wall intersections of even complex Manhattan world rooms given unoccluded observations. Previtali et al. (Previtali et al., 2014) and Murali et al. (Murali et al., 2017) use a similar method for their detected wall lines. They also use ad-hoc blueprints of the building using the floor and ceiling geometry to better evaluate the potential intersections. Both methods process single rooms and target the individual wall faces. In contrast, our method targets entire floors and focuses on volumetric walls. Ambrus et al. (Ambrus et al., 2017) successfully extend cell decomposition to entire floors but still targets individual wall faces in contrast to our method. Another approach that simultaneously reconstructs multiple rooms is the method of Turner et al. (Turner, Zakhor, 2014). They generate a 2D Delaunay mesh based on their partial wall geometry and use graph-cuts to assign the triangles to different rooms based on a set of seed nodes. They then merge the naked edges of each grown patch, which results in a generated floor plan. While their results are promising for their application, their approach does not operate on volumetric wall objects and they do not attempt to create watertight geometry. Cell decomposition is also used in a variety of other applications such as roof reconstruction (Kada, McKinley, 2009), building hull extraction, and wall reconstruction (Budroni, Boehm, 2010, Michailidis, Pajarola, 2016). An interesting feature is that the topology and geometry is constructed simultaneously. However, in this research, we consider both steps separately to compute the best fit partial wall geometry before evaluating the topology.

There are several approaches that closely align with our work which are discussed in (Ochmann et al., 2019). In comparison 
to our approach, Mura et al. (Mura et al., 2014, Mura et al., 2016) use $2 \mathrm{D}$ cell decomposition on an entire floor and decide which intersections are valid based on the floor and ceiling information. Furthermore, they reconstruct volumetric wall entities similar to our BIM wall outputs. We extend their work by considering connections between more complex centrelines of walls such as arcs and polylines. Next, there is Oesau et al. (Oesau et al., 2014). They perform an indoor scene reconstruction based on a 3D cell decomposition. They specifically target complex geometry and succeed in creating a watertight model of the faces of the interior of buildings. We also operate in 3D but focus on volumetric parametric entities instead of BREP geometry. In their recent study, Ochmann et al. (Ochmann et al., 2019) proposed a fully automated volumetric reconstruction based on 3D cell decomposition, with very promising results. We propose a similar approach but both allow more types of connections (such as the shortest euclidean distance) and we use more complex centrelines as input. The goal is to not only reconstruct straight walls with proper observations but also deal with the small percentage of complex wall configurations.

\section{METHODOLOGY}

The proposed wall topology reconstruction operates as follows. We start from the partial walls and propose a set of candidate connections. Based on minimal adjustments, the most logic set of connections is retained. Given the nodes of each connection, the wall centrelines of the walls are adjusted. The method operates on IfcWallStandardCase objects, which is the desired deliverable of many BIM applications. To this end, it was implemented in both Revit and Rhino (Fig. 1). The consecutive steps are discussed in detail in the following paragraphs.

\subsection{Preprocessing}

As previously stated, several preparatory steps of the presented approach are performed in prior work. First, the unstructured point cloud is represented as a voxel octree after which planar patches are extracted using an efficient parallel processing region growing (Bassier et al., 2017). To reduce the degree of oversegmentation, a first associative Conditional Random Field is employed to merge similar clusters that got separated due to data tiling. Next, the planar patches are subjected to a reasoning framework that computes class labels for each patch. A pre-trained Random Forests model is used for the classification (Bassier et al., 2018b). This classification model is chosen for its robustness against high variance datasets as is the case with characteristics of building geometry observations due to wall detailing, openings, arched walls and so on. The result is a set of labeled segments that replaces the point cloud representation of the building. Following, the segments are clustered into groups that represent the individual walls. A second Conditional Random Field exploiting local and contextual information is employed to compute the most likely assignment of the wall segments (Bassier, Vergauwen, 2019). In contrast to the existing literature, the wall clustering does not solely rely on coplanarity or parallelity for its clustering, thus again making it more robust against the high variance of wall observation's characteristics. The result is a set of clustered wall mesh segments that represents all the available observations of each wall. This is a highly reliable and accurate representation of the observed structure but it is incomplete due to occlusions and thus can only serve as a static model. Therefore, in a final step, partial LOD200 (BIMForum, 2016) wall geometry is established based on the clustered segments that represents the basic parametric geometry of the walls (Bassier et al., 2018a). The 2D centrelines of these entities serve as the input for the presented method.

\subsection{Topology estimation}

The estimation of the topology is performed iteratively and consists of three main phases (Fig. 2). First, an eligible seed $p_{i}$ is established at the end of a centreline. Next, the potential connections $C$ are defined for $p_{i}$ based on a set of neighbors $Q$. Iteratively, the best fit set of connections is withheld until all possible connections are resolved. In a final step, the new sections of centrelines are created and joined with the partial wall geometry to form a more faithful BIM. Each step is discussed in detail below.

Seed and neighbors The seed $p_{i}$ and its neighbors $Q$ are found as follows. The endpoint of a centreline is considered a seed if it does not intersect with another centreline. For a seed $p_{i}$ of a centreline $l_{i}$, the centrelines $N$ are found that contain a point $q_{i}$ lying within a user defined threshold distance $t_{d}$ of $p_{i}$ (Eq. 1). This distance is set equal to the maximum gap a wall is allowed to span over to make a connection and is application dependent.

$$
\begin{aligned}
& q_{j}=\left(p_{i} \in l_{i} \wedge q_{j} \in l_{j}: \operatorname{argmin}_{q_{j}}\left(\left\|p_{i}-q_{j}\right\|\right)\right) \\
& Q=\left\{q_{j} \mid \forall l \in L \backslash\left\{l_{i}\right\} \wedge p_{i} \in l_{i} \wedge q_{j} \in l_{j}:\left\|p_{i}-q_{j}\right\| \leq t_{d}\right\} \\
& N=\left\{l \in L \mid \forall q_{j} \in Q: l \cap q_{j}\right\}
\end{aligned}
$$

where the smallest euclidean distance $\left\|p_{i}-q_{j}\right\|$ is observed to retain a neighboring centreline. $Q$ is the set of the possible connection points of $p_{i}$ and $N$ is the set of curves to which $Q$ belongs.

Candidate connections Given the $N$ and $Q$, several connections can be made between $p_{i}$ and every $q_{j} \in Q$. In contrast to the literature, we are able to process both straight edges, arcs and polylines. Additionally, we define multiple types of connections (Fig. 2): the intersection between the centrelines $l_{i}$ and $l_{j}$, the intersection between the normals at $p_{i}$ and $q_{j}$ and finally also the connection with the shortest euclidean distance $\overrightarrow{p_{i} q_{j}}$ (Eq. 2).

$$
\begin{aligned}
& U=\left\{u_{i j} \mid \forall l_{j} \in N \wedge p_{i} \in l_{i}: l_{i} \cap l_{j}\right\} \\
& V=\left\{v_{i j} \mid \forall q_{j} \in Q \wedge p_{i} \in l_{i}: \overrightarrow{n_{p_{i}}} \cap \overrightarrow{n_{q_{j}}}\right\}
\end{aligned}
$$

where $U$ and $V$ are the intersection points of respectively the functions themselves and their normals at $p_{i}$ and $q_{j}$. This results in a set $c \in C$ of three types of potential connections between $p_{i}$ and its neighboring centrelines (Eq. 3).

$$
c \in C= \begin{cases}\text { Intersection: } & \left\{\overrightarrow{p_{i} u_{i j}}, \overrightarrow{q_{j} u_{i j}}\right\} \\ \text { Orthogonal connection: } & \left\{\overrightarrow{p_{i} v_{i j}}, \overrightarrow{q_{j} v_{i j}}\right\} \\ \text { Shortest connection: } & \overrightarrow{p_{i} q_{j}}\end{cases}
$$



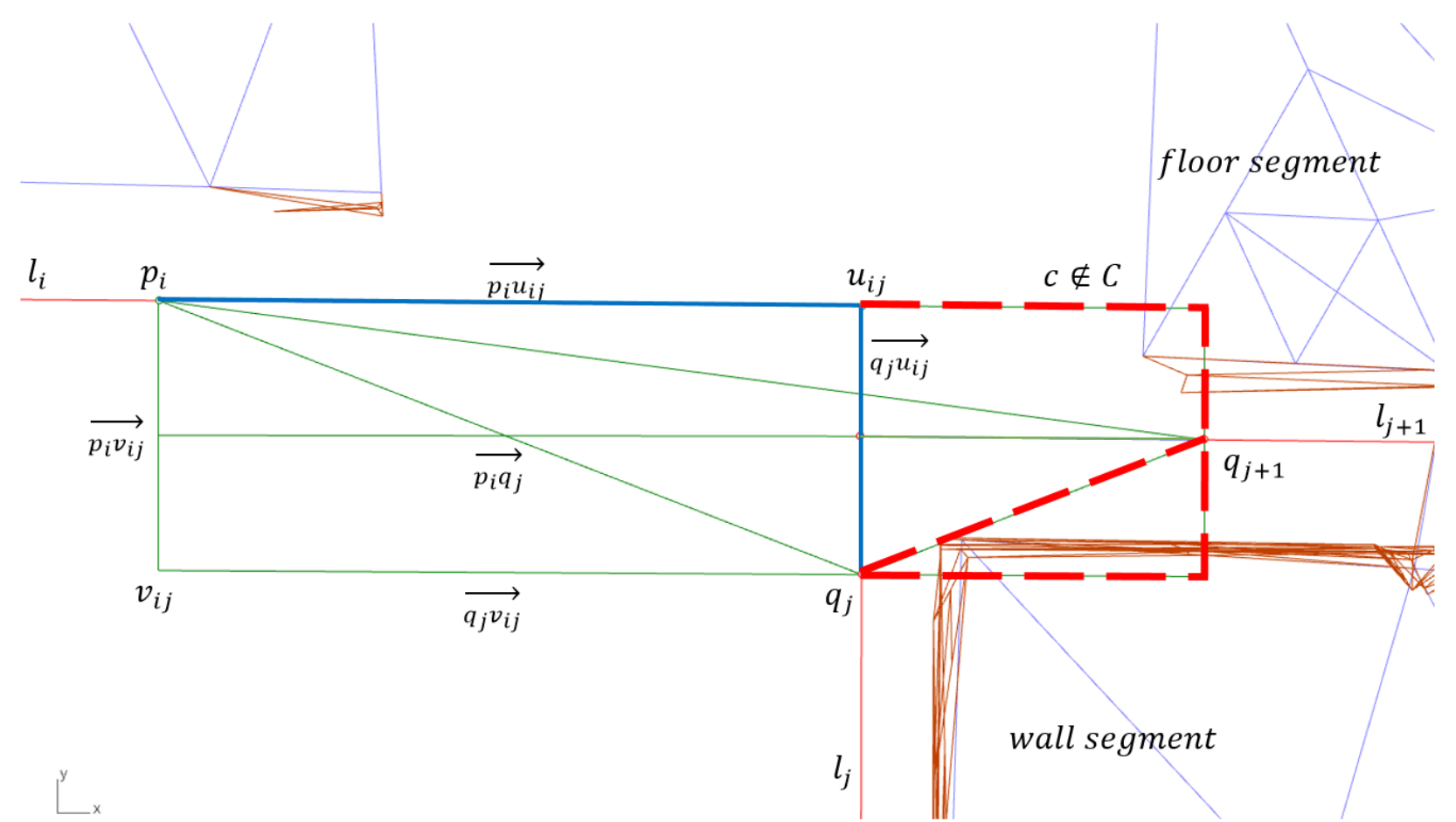

(a) First iteration: $\left\{\overrightarrow{p_{i} u_{i j}}, \overrightarrow{q_{j} u_{i j}}\right\}$ is prioritized over $\left\{\overrightarrow{p_{i} v_{i j}}, \overrightarrow{q_{j} v_{i j}}\right\}$ and $\overrightarrow{p_{i} q_{j}}$.

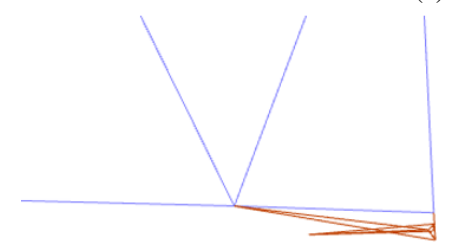

$\underline{l_{j}}$

$l_{j}$

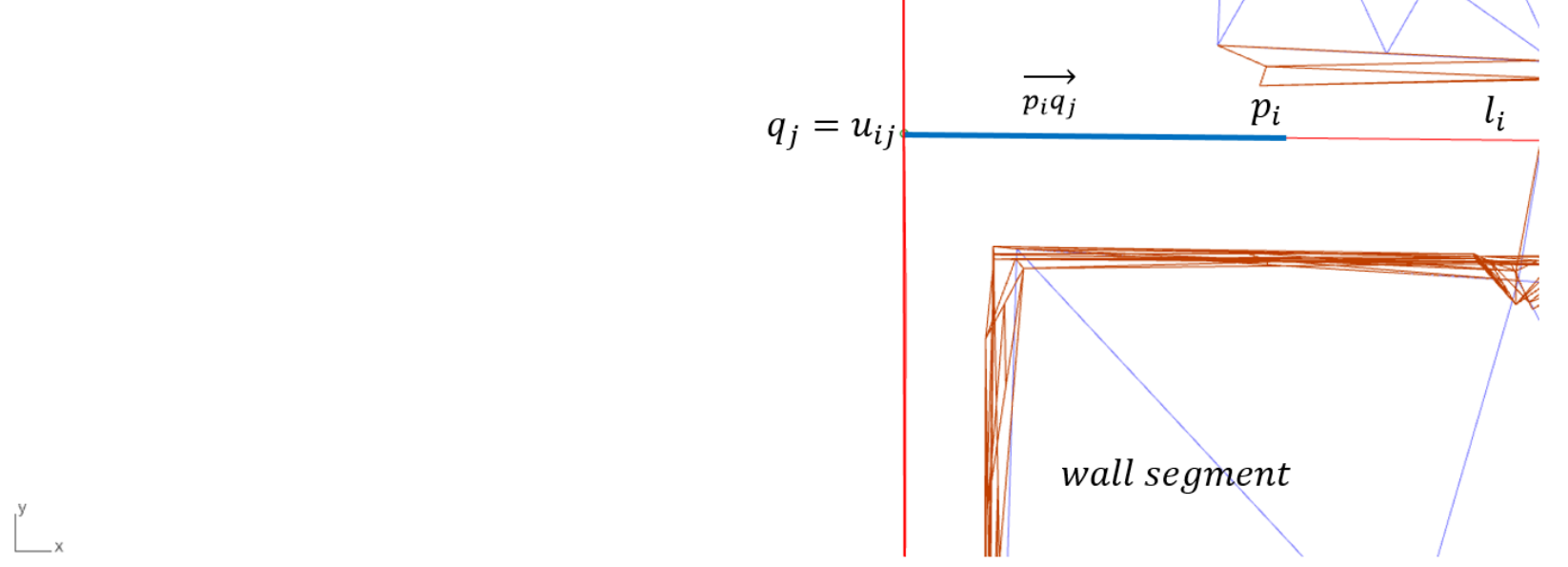

(b) Second iteration: $q_{i}=u_{i j}$, and thus the orthogal connection $\left\{\overrightarrow{p_{i} u_{i j}}\right\}$ is prioritized.

Figure 2. Example wall topology reconstruction of a 3 wall configuration depicting the nearby wall (brown) and floor (purple) segments, the centrelines (red), the potential connections $C$ (green lines), the rejected connections (red dotted lines). The blue lines are the reconstructed centrelines in subsequent iterations to complete the wall topology.

However, not every possible connection should be considered. It is our assumption that possible connections should not intersect with floor or ceiling geometry as this violates building logic. Also, the connection should not intersect with wall segments. Therefore, $C$ is conditioned to not intersect with any extruded floors, ceilings and walls (Eq. 4).

$$
C^{\prime}=C \backslash\{\text { floors, ceilings, walls }\}
$$

Centreline creation The best suited connection is chosen in a pairwise manner. During each iteration, a maximum of $2 c \in$ 
$C$ is selected to connect two centrelines. The two centrelines are decided based on the most valid potential connections in between them. Subsequently, a hierarchical method is employed prioritizing valid intersecting connections $\left\{\overrightarrow{p_{i} u_{i j}}, \overrightarrow{q_{j} u_{i j}}\right\}$ over orthogonal connections $\left\{\overrightarrow{p_{i} v_{i j}}, \overrightarrow{q_{j} v_{i j}}\right\}$ and finally over the shortest euclidean distance $\overrightarrow{p_{i} q_{j}}$. When a connection is established, $l_{i}$ and $l_{j}$ are extended with the chosen segments. Once the segments are created, the process is repeated for the next $p_{i}$ until no more eligible seeds can be found. The major advantage of the presented method is that it also deals with complex wall intersections with multiple walls and types of centrelines.

\subsection{Wall creation}

The result of the topology reconstruction is a set of adjusted wall centrelines. Using Rhino.Inside (Robert McNeel \& Associates, 2019), additional native Revit LOD200 walls are constructed to update the existing model. The result is topologically sound model in frequently used formats that can be used in wide variety of applications.

\section{EXPERIMENTS}

The proposed algorithm can be successfully used in Revit and Rhino using the Rhinocommon API and Rhino.Inside. Figure 1 shows an example of the interface between both software and the automatically reconstructed topology. Both the estimation of the candidate connections and the topology adjustment were performed fully automatically. Several examples are presented to test the performance of the algorithm. The tests include 2-wall connections and also more complex 3-wall connections for different configuration of lines, arcs and polylines (Table.1). Each case is simulated with centrelines from a number of mesh observations which is the output from our previous work (Bassier, Vergauwen, 2019). The centrelines represent realistic conditions with near-parallel functions, different configurations and different types of functions. The automatically reconstructed geometry is compared to the manually designed walls.

The wall topology reconstruction computed a proper solution for most wall scenarios. There are of course the more complicated wall configurations that, without the support of additional information, can have multiple outcomes. However, the presented centrelines closely align with the manually constructed topology. Several weaknesses still remain in the method. First of all, without the presence of observed ceiling, floor and other wall geometry, the method's reliability can be compromised. While prioritizing intersecting connections gives promising results even in occluded scenarios, we cannot know for certain whether this is in fact the correct topology. A second aspect is the choice of the seed $p_{i}$, which currently is based on the centreline length. This generally leads to a proper wall configuration but not necessarily to the proper walltype. Especially in more complex scenarios where a first adjustment is already computed, there might be confusion about which walltype should be chosen for the secondary and tertiary wall segments. We state that the model should still be validated by a user to ensure that the proper walltype is chosen for the additional centrelines. Finally, there is also the assumption of function continuity for the intersecting curves. As the distance threshold increases, the extrapolation of functions (especially for arcs and polylines) become increasingly unreliable. This problem is again tied to the occlusions in the model which should be avoided wherever possible.

\section{CONCLUSION}

This paper presents an semi-automated method to reconstruct the wall topology of Building Information Models from a set of partial centrelines that form the basis of parametric wall geometry. The method takes as input 2D curves including lines, arcs and polylines originating from previous work and outputs Native Revit and Rhino wall extensions. Given the centrelines of a set of LOD200 partial walls, the method computes a set of potential connections between the walls including intersections, orthogonal connections and the shortest euclidean distance between the curves. Iteratively, the best fit connection is withheld by prioritizing intersections over orthogonal and euclidean connections. Additionally, the potential connections are conditioned to not intersect with wall, floor and ceiling segments to avoid false positives. The result is a set of wall adjustments that form a more faithful as-built BIM representation.

The experiments indicate that the used method is a promising topology reconstruction framework. The proposed wall adjustments are similar to what human modelers would prefer and the method deals with complex curves and configurations which can significantly speed up the manual modeling process. Additionally, the method can also operate on existing models as long as a set of centrelines is extracted from the existing walls. The method currently solely operates on parametric walls based on $2 \mathrm{D}$ centrelines. In future work, the wall topology will be extended to deal with more complex wall geometry to further expand the automation of as-built BIM reconstruction.

\section{ACKNOWLEDGMENTS}

This project has received funding from the European Research Council (ERC) under the European Union's Horizon 2020 research and innovation programme (grant agreement 779962) and the Geomatics research group of the Department of Civil Engineering, TC Construction at the KU Leuven in Belgium.

\section{REFERENCES}

Ambrus, R., Claici, S., Wendt, A., 2017. Automatic Room Segmentation From Unstructured 3-D Data of Indoor Environments. IEEE Robotics and Automation Letters, 2(2), 749-756.

Bassier, M., Klein, R., Van Genechten, B., Vergauwen, M., 2018a. Ifcwall Reconstruction from Unstructured Point Clouds. ISPRS Annals of Photogrammetry, Remote Sensing and Spatial Information Sciences, IV-2, 33-39. https://www.isprs-ann-photogramm-remotesens-spatial-inf-sci.net/IV-2/33/2018/.

Bassier, M., M, B., Van Genechten, B., M, V., 2017. Octree-Based Region Growing and Conditional Random Fields. The International Archives of the Photogrammetry, Remote Sensing and Spatial Information Sciences, Volume XLII-2/W8, 2017 5th International Workshop LowCost $3 D$-Sensors, Algorithms, Applications, XLII(November), 28-29.

Bassier, M., Van Genechten, B., Vergauwen, M., Genechten, B. V., Vergauwen, M., 2018b. Classification of sensor independent point cloud data of building objects using random forests. Journal of Building Engineering, 110. https://doi.org/10.1016/j.jobe.2018.04.027. 
Table 1: Result of the automated topology estimation given the reconstructed centrelines. Each row represents the fitting results for the Line, Arc and Polyline for a 2 Line and a 3 Line configuration. The brown lines show the wall segments, the surrounding floor and ceiling segments are shown in purple. The potential connection $\mathrm{C}$ are shown as green lines and the final connections are show as blue lines. The green dots are the collection of intersection points and $\mathrm{p}$ and $\mathrm{q}$

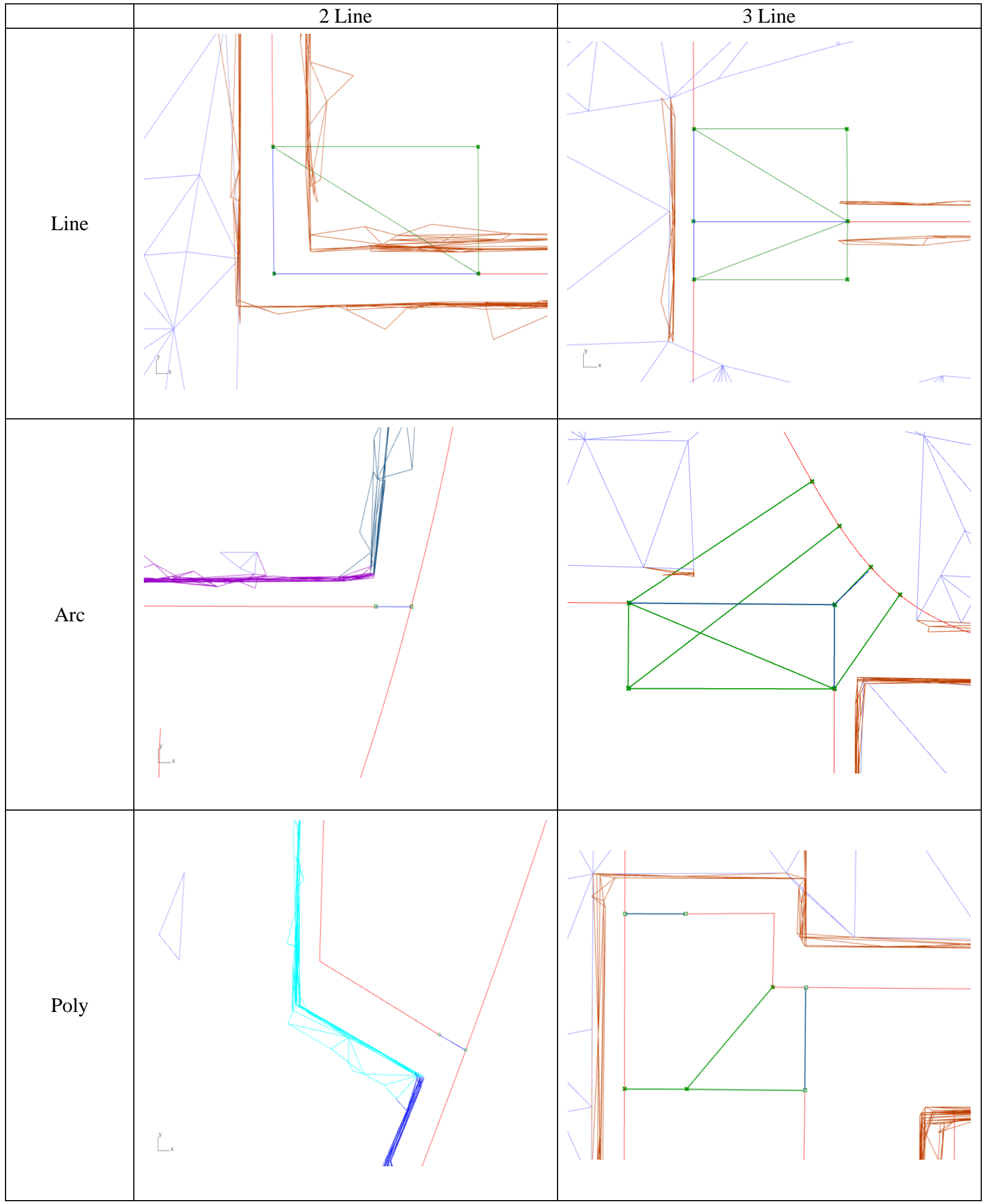


Bassier, M., Vergauwen, M., 2019. Clustering of Wall Geometry from Unstructured Point Clouds Using Conditional Random Fields. Remote Sensing, 42(2/W9), 101108 .

BIMForum, 2016. Level of Development Specification. Technical report, BIMFORUM.

Bosché, F., Guillemet, A., Turkan, Y., Haas, C. T., Haas, R., 2014. Tracking the Built Status of MEP Works: Assessing the Value of a Scan-vs-BIM System. Journal of Computing in Civil Engineering, 28(4), 5014004-5014013. http://ascelibrary.org/doi/abs/10.1061/(ASCE)CP.19435487.0000343.

Bosché, F., Turkan, Y., Haas, C., 2013. Tracking the Built Status of MEP Works : Assessing the Value of a Scan-vs . -BIM System. Journal of Computing in Civil Engineering. http://dx.doi.org/10.1016/j.autcon.2014.05.014.

Budroni, A., Boehm, J., 2010. Automated 3D Reconstruction of Interiors from Point Clouds. International Journal of Architectural Computing.

Gimenez, L., Robert, S., Suard, F., Zreik, K., 2015. Automatic reconstruction of $3 \mathrm{D}$ building models from scanned 2D floor plans. Automation in Construction, 63(2016), 4856. http://dx.doi.org/10.1016/j.autcon.2015.12.008.

Hichri, N., Stefani, C., Luca, L. D., Veron, P., 2013. Review of the As-Built Bim Approaches. 3D-ARCH 2013 $3 D$ Virtual Reconstruction and Visualization of Complex Architectures, XL-5/W1(February).

Kada, M., McKinley, L., 2009. 3D Building Reconstruction from LIDAR based on a Cell Decomposition Approach. CMRT09: Object Extraction for 3D City Models, Road Databases and Traffic Monitoring - Concepts, Algorithms and Evaluation, XXXVIII, 47-52.

Kavanaugh, J. C. K. S. P. E. B., 2013. Client-Guide-to3D-Scanning-and-Data-Capture. Technical report, Building Information Modelling Task Group.

Michailidis, G. T., Pajarola, R., 2016. Bayesian graph-cut optimization for wall surfaces reconstruction in indoor environments. Visual Computer, 1-9.

Mura, C., Mattausch, O., Jaspe Villanueva, A., Gobbetti, E., Pajarola, R., 2014. Automatic room detection and reconstruction in cluttered indoor environments with complex room layouts. Computers \& Graphics, 44, 20-32.

Mura, C., Mattausch, O., Pajarola, R., 2016. Piecewiseplanar Reconstruction of Multi-room Interiors with Arbitrary Wall Arrangements. Computer Graphics Forum, 35(7), 179-188.

Murali, S., Speciale, P., Oswald, M. R., Pollefeys, M., 2017. Indoor Scan2BIM: Building information models of house interiors. IEEE International Conference on Intelligent Robots and Systems, 2017-Septe.

Nguyen, A., Le, B., 2013. 3D point cloud segmentation : A survey 3D. 2013 6th IEEE Conference, 225-230.

Ochmann, S., Vock, R., Klein, R., 2019. Automatic reconstruction of fully volumetric 3D building models from oriented point clouds. ISPRS Journal of Photogrammetry and Remote Sensing.
Oesau, S., Lafarge, F., Alliez, P., 2014. Indoor scene reconstruction using feature sensitive primitive extraction and graph-cut. ISPRS Journal of Photogrammetry and Remote Sensing, 90, 68-82.

Patraucean, V., Armeni, I., Nahangi, M., Yeung, J., Brilakis, I., Haas, C., 2015. State of research in automatic as-built modelling. Advanced Engineering Informatics, 29, 162-171.

Previtali, M., Barazzetti, L., Brumana, R., Scaioni, M., 2014. Towards automatic indoor reconstruction of cluttered building rooms from point clouds. ISPRS Annals of Photogrammetry, Remote Sensing and Spatial Information Sciences, II-5(June), 281-288. http://www.isprs-ann-photogramm-remote-sens-spatialinf-sci.net/II-5/281/2014/.

Robert McNeel \& Associates, 2019. Rhino.Inside.

Tah, J., Oti, A., Abanda, F., 2018. A state-of-the-art review of built environment information modelling (BeIM). Organization, Technology and Management in Construction: an International Journal, 9(1), 1638-1654.

Turner, E., Zakhor, A., 2014. Floor Plan Generation and Room Labeling of Indoor Environments from Laser Range Data. GRAPP, International Joint Conference on Computer Vision, Imaging and Computer Graphics Theory and Applications, 1-12.

Valero, E., Adán, A., Cerrada, C., 2012. Automatic method for building indoor boundary models from dense point clouds collected by laser scanners. Sensors (Switzerland), 12(12).

Volk, R., Stengel, J., Schultmann, F., 2014. Building Information Modeling (BIM) for existing buildings - Literature review and future needs. Automation in Construction, 38, 109-127. http://dx.doi.org/10.1016/j.autcon.2013.10.023.

Xiong, X., Adan, A., Akinci, B., Huber, D., 2013. Automatic creation of semantically rich 3D building models from laser scanner data. Automation in Construction, 31, $325-337$. 